\title{
Editorial note to: Brandon Carter, Large number coincidences and the anthropic principle in cosmology
}

\author{
George F. R. Ellis
}

Published online: 27 September 2011

(C) Springer Science+Business Media, LLC 2011

Keywords Cosmology - Confrontation of cosmological theories with observational data · Anthropic principle · World ensemble · Golden Oldie Editorial

The anthropic principle is one of the most controversial proposals in cosmology. It relates to why the universe is of such a nature as to allow the existence of life. This inevitably engages with the foundations of cosmology, and has philosophical as well as technical aspects. The literature on the topic is vast- the Carter paper reprinted here [10] has 226 listed citations, and the Barrow and Tipler book [3] has 1,740. Obviously I can only pick up on a few of these papers in this brief review of the impact of the paper.

While there were numerous previous philosophical treatises on the topic, stretching back to speculations about the origin of the universe in ancient times (see [3] for a magisterial survey), scientific studies are more recent. A well known early one was by biologist Alfred Russell Wallace, who wrote in 1904: "Such a vast and complex universe as that which we know exists around us, may have been absolutely required ... in order to produce a world that should be precisely adapted in every detail for the orderly development of life culminating in man" [50]. But that was before modern cosmology was established: the idea of the expanding and evolving universe was yet to come.

Following on Weyl's speculations on the fundamental physical constants in 1919, and Eddington's work from 1923 on (summarised in his book [25]) hoping to explain the numerical values of these constants, ${ }^{1}$ Dirac's ruminations on the large number coincidences between these constants [22] seem to have set the ball rolling. He suggested

\footnotetext{
${ }^{1}$ See pp. 224-231 of [3] for detailed references.
}

The republication of the original paper can be found in this issue following the editorial note and online via doi:10.1007/s10714-011-1258-7. 
[23] that these coincidences in the evolving universe had to be explained by a time varying gravitational constant, else they would only be true at one cosmic epoch.

Robert Dicke [21] noted in 1961 that Dirac's relation could be a selection effect related to the times when observers could exist, this in turn being related to the values of the fundamental constants of physics which determine the lifetime of main sequence stars. Dicke's paper makes an explicit anthropic statement in the cosmological context, based on an analysis of the parameters affecting stellar lifetimes: " $T$ [the age of the universe] is not a random choice from a wide range of possible choices but is limited by the criteria for the possible existence of physicists".

Brandon Carter, then a postdoc at Cambridge University, mused on these topics, being inspired to do so first by the discussion of Dirac's work in Bondi's classic book Cosmology [4], and then by Dicke's paper [21]. In 1967 he wrote a preprint [8] systematically investigating the causal links between these constants and both astronomical and physical phenomena, focusing first on the mass $m_{N}$ of the nucleon and its relation to the electric charge $e$ and the mass of the electron $m_{e}$, and then including three more sufficient to determine ordinary everyday physics (the strong interaction coupling constant $g_{S}$, the pion mass $m_{\pi}$, and the mass difference $\Delta_{N}$ between the neutron and the proton) and three relating to more exotic processes (the muon mass $m_{\mu}$, the electromagnetic mass splitting $\Delta_{\pi}$ of the pion, and the weak coupling constant $g_{W}$ ). He commented on some remarkable relations between these parameters (equations (6)-(8) of [8]). He calculated many astrophysical consequences of these numbers, but did not make any explicit anthropic link. A second preprint in 1968, that was less widely circulated, made the anthropic link to their values in the context of an ensemble of universes. ${ }^{2}$ This then opened the way for his conference talks on 21 February, 1970 at a Clifford Centenary meeting in Princeton, ${ }^{3}$ and at an IAU Symposium honouring Copernicus's 500th birthday held in Cracow from September 10 to 17, 1973 (both talks being at the invitation of John Wheeler). The latter talk was published in the symposium proceedings in 1974 [10], which we reprint here as a Golden Oldie. This definitively opened up the topic of the anthropic principle as a subject for scientific discussion in the context of present day cosmological theory. It was contemporaneous with a paper by Collins and Hawking [18], "Why is the universe isotropic?", that raised the same issue in the specific context of Bianchi cosmologies. The authors were all at the same institution, so it was the subject of discussion between them at that time; Carter's work is referenced in the Collins and Hawking paper, which in turn is referenced in Carter's 1974 paper.

The Collins and Hawking paper was a specific application to the case of Bianchi cosmologies, showing that only some Bianchi classes would be suitable for life to develop. It states "One cannot explain the isotropy of the universe without postulating special initial conditions ... we have to face the awkward question, why is the universe isotropic?" Like Carter they consider an ensemble of universes. After explaining that only in Bianchi universes that tend to become isotropic are galaxies likely to form, the paper states

\footnotetext{
2 This preprint is referred to in the Collins and Hawking paper ([18], p. 319).

3 Carter [9]; Referred to in [16].
} 
SAP (Collins and Hawking 1973): "Since it would seem that existence of galaxies is a necessary condition for the development of intelligent life, the answer to the question 'why is the universe isotropic?' is 'because we are here' ".

This statement appears to invert the expected direction of causality, and so led to some suspicion about the anthropic principle in many circles; however it in fact was to be seen as an explanatory proposal in the context of a multiverse (which was mentioned earlier in the paper).

The physics based approach by Carter [10] in his paper opened up much interest in the Anthropic Principle as a scientific method of explanation. In addition to the mass of the proton $m_{p}$, the mass of the electron $m_{e}$ and the electric charge $e$, like Dicke he introduced the Hubble expansion rate $H$ (closely related to the age of the universe $\simeq H^{-1}$ ), and related these to other cosmological parameters and conditions required for the existence of observers. He characterised the Weak Anthropic Principle (used by Dicke in [21]) as follows:

WAP (Carter 1974): "We must be prepared to take account of the fact that our location in the universe is necessarily privileged to the extent of being compatible with our existence as observers".

Thus it is seen as an observational selection effect in a universe that is biofriendly; it relates to where and when we can exist in such a universe ("location" refers to time as well as space), and is to be seen as a contrast to the Copernican principle (all places are to be regarded as equivalent). He then proposed the Strong Anthropic Principle, relating to severe restrictions on the fundamental parameters of the universe itself if life is to exist:

SAP (Carter 1974): "The universe (and hence the fundamental parameters on which it depends) must be such as to admit the creation of observers within it at some stage".

This sounds like the Collins and Hawking statement. But this was intended as a prediction, not an explanation; it took the existence of observers for granted, and made deductions on that basis. However he then introduced the idea that an ensemble of universes was necessary in order that the conditions for life to exist could be explained in a probabilistic way:

SAP+ (Carter 1974): "It is of course philosophically possible_-as a last resort, when no stronger physical argument is available - to promote a prediction based on the strong anthropic principle to the status of an explanation by thinking in terms of a "world ensemble'".

Selection then takes place in the context of the multiverse, selecting out biofriendly universes from the rest, and hence explaining why the nature of physics in such universes is friendly to life. This is the most common interpretation of the principle at the present time.

The topic was developed further in an important paper by Carr and Rees [16], broadening the set of relationships between the constants considered. The abstract of that paper states, "The basic features of galaxies, stars, planets and the everyday 
world are essentially determined by a few microphysical constants and by the effects of gravitation. Many interrelations between different scales that at first sight seem surprising are straightforward consequences of simple physical arguments. But several aspects of our Universe-some of which seem to be prerequisites for the evolution of any form of life-depend rather delicately on apparent 'coincidences' among the physical constants." A similar discussion was given later by Davies [19]. This suggests that there is something needing explaining, unless these bio-friendly relations were just coincidences - but scientific progress often lies in showing that what at first seem like coincidences are in fact necessary relations.

The topic was then picked up in a Royal Society discussion meeting on the constants of nature organised in 1983 by W H McCrea, where Carter stated the strong principle as follows:

SAP (Carter 1983): "Our mere existence as intelligent observers imposes restrictions not merely on our situation, but even on the general properties of the universe including the values of the fundamental parameters". [11]

This version again is not an explanation, but a deduction from the fact of our existence. Carter explained that this did not imply that life was probable; he predicted that the occurrence of observers would be rare, even on environmentally favorable planets such as ours.

The topic was then presented in an encyclopaedic way in the book by Barrow and Tipler [3], covering old design arguments, modern teleology, various Anthropic principles, and their relation to quantum mechanics and biochemistry. This book became a major reference in the area and made it widely known. It interpreted the Weak Anthropic Principle as a selection effect: "any cosmological observations made by astronomers are biassed by an all-embracing selection effect: our own existence" ([3], pp. 15-16), but this selection effect is interpreted in a multiverse setting (p. 19). Their version of the SAP (p. 21) is essentially the same as Carter's 1974 version. They introduced two further versions: Wheeler's controversial Participatory Anthropic Principle (PAP), based on his interpretation of quantum theory: "Observers are necessary to bring the universe into being"; and their own Final Anthropic Principle:

FAP (Barrow and Tippler 1986): "Intelligent information-processing must come into existence in the Universe, and, once it comes into existence, it will never die out".

These two inclusions somewhat discredited an otherwise very fine volume. In his famous review of the book, Martin Gardner [30] ridiculed the FAP by quoting the last two sentences of the book, which he characterized as representing the Completely Ridiculous Anthropic Principle (CRAP). These versions (PAP and FAP) have now faded into obscurity.

The publication of this book opened the floodgates for more philosophically based discussions as well as popular presentations (e.g. [24,39,46,43,33,20,5]). An excellent survey article was written by Balashov [1], with extensive references up to 1991. The more the issue of fine tuning was investigated, the more pressing it became. Rees for example [43] argued that anthropic reasoning is needed to explain the values of six constants required for life to exist: 
- $N \simeq 10^{36}$ : the strength of the electrical forces that hold electrons and protons together to form atoms, divided by the force of gravity between them;

- $\epsilon \simeq 0.007$ : defining how strongly atomic nuclei bind together;

- $\Omega_{m} \simeq 0.3$ : the actual density of the universe expressed in terms of the critical density required for it to eventually recollapse in the future, if there is no cosmological constant;

- $\Omega_{\Lambda} \simeq 0.7$ : the energy density of the cosmological constant, similarly expressed as a dimensionless ratio;

- $Q \simeq 10^{-5}$ : the fractional density of the fluctuations in the early universe that were seeds of structure formation via gravitational attraction;

- $D=3$ : the number of spatial dimensions in our world (at macroscopic scales).

The constants $N, \epsilon$ and $D$ govern the fundamental interactions of physics; $\Omega_{m}, \Omega_{\Lambda}$ and $Q$ govern the expansion of the universe and the growth of structures in it.

Two comments on this: first, these are not the only parameters that have to be fine tuned, for example the proton-neutron mass difference is not listed here. The interactions between physical constants are complex, and indeed it is not clear which should be thought of as fundamental (see $[33,2,48,49]$ ); for example these parameters are not precisely the same as those discussed by Carter $[8,10]$, although they are related. Second, one must regard the phrase 'fine tuning' with caution. What is meant here is that the range of parameter values allowing life is a restricted part of the possibility space for the parameter, not always that it has to lie in a narrow range of values (though that is often indeed the case). Martin Rees comments, "Some authors incorrectly claim that there is 'fine tuning' in $G$ (or what I called $N$ in my book). Of course there isn't; the essential anthropic requirement is just that $N$ should be very large. Indeed, if it were 10 to 100 times bigger (with longer-lived and bigger stars and planets, etc) there might have been even more space and time for complex evolution." This is an important restriction: a priori, it need not have been the case.

Although the evidence for fine tuning in this sense was accumulating, the anthropic principle project was initially not widely accepted: it was regarded as a philosophical oddity in cosmology, not really related to physics. It became much more widely accepted as a result of three developments.

Firstly, there was a growing appreciation of the major problem of the magnitude of the cosmological constant, when considered as the vacuum energy of quantum fields. Simple quantum field theory calculation suggests it should be many orders of magnitude larger than the astronomically determined value [52]. This is a major problem for theoretical physics, because this prediction arises from a plausible combination of two of our most successful theories, namely general relativity and quantum field theory. This has crucial implications for astrophysics because a value much larger than observed would prevent galaxy formation, and hence would result in a lifeless universe. A multiverse solution seemed almost the only way out [51,52].

Secondly, the proposal of chaotic inflation [40] arose as a variant of Guth's inflationary universe proposal, and this seemed to provide a natural setting for existence of many disconnected expanding universe domains that could have different effective physical constants in each of them. This could reasonably be considered a physical 
mechanism for realization of a 'multiverse', as originally envisaged by Carter [10] and Collins and Hawking [18].

Thirdly, the proposal of the landscape of string theory arose as a physics setting within which variation of effective constants might naturally arise $[44,45]$. The huge number of string vacua posited in that theory provided a setting whereby the effective constants of physics could be different in different domains such as occur in chaotic inflation.

Together these ideas gave a coherent explanation of an otherwise troubling issue: why does the cosmological constant have a small value that allows life to exist? Many different types of physics occur in the different universes in a multiverse; because we are here to observe events around us, we necessarily live in one of the universes in the multiverse where the values of fundamental constants are such as to allow life to exist.

Once one has this theoretical setting, one can apply the same explanation to any other of the 'fundamental' physical constants that need fine tuning in order that life can exist, such as those mentioned by Rees [43]: they too can arise from a deeper theory (M-Theory) that allows each of them to have values that vary with position in a multiverse. One can even suggest that in this way, the anthropic principle is indispensible for making sense of the hypothesized landscape of superstring theory (Brandon Carter, private communication). However one should note that the basic mechanism for eternal inflation does not of itself cause physics to be different in each domain in a multiverse. This supposed variation is not a necessary conclusion arising from the straightforward conjunction of the physics of chaotic inflation and $\mathrm{M}$ theory; but it can be the outcome if one extends those theories by adding extra assumptions. Thus the proposal is speculative both in terms of assuming correctness of the landscape of string theory, which is contested within the string theory community, and these extra assumptions.

Proponents of the SAP in its explanatory form include Andrei Linde, Martin Rees, Stephen Weinberg, and Leonard Susskind, but others such as Roger Penrose, David Gross, and Lee Smolin disagree. Various authors (including Aguirre, Bostrom, Carr, Carter, Davies, Dimopoulos, Donoghue, Hartle, Hogan, Kallosh, Linde, Page, Rees, Smolin, Stoeger, Susskind, Vilenkin, Weinberg, and Wilczek) summarize their views on anthropic proposals in [6]. A recent review of the principle by Carter is [12].

Various criticisms have been made of the proposal; I summarize them as follows:

First, Carter [12] has regretted his use of the word "anthropic", because it conveys the impression that the principle involves only humans, rather than general intelligent observers; others have commented that the term is misleading because the usual calculations have nothing directly to do with humans, but refer only to complex atoms or stars and planets. However these remarks do not detract from the fact that the principle as commonly used relates to real restrictions that will plausibly apply to existence of any materially-based form of intelligent life, and hence is indeed an interesting topic of discussion. Nevertheless if one is to seriously contemplate the relation of physics to the origin of life, one does need to relate the theory to biological realities $[11,12,37,15]$.

Second, there has been criticism that the word "principle" is not justified in describing what are in fact just selection effects, or indeed perhaps tautologies. However selection effects play a crucial role in science, and are far from trivial; and in particular, 
examining the consequences of changes in the fundamental constants for existence of life is a useful and complex task. It certainly does succeed in showing that the physical conditions required for the existence of life are very special relative to what might conceivably have been $[3,43,1,27]$.

Third, the explanatory form of SAP has been criticized by many physicists as simply giving up on physical explanation. Penrose states it thus" : "It tends to be invoked by theorists whenever they do not have a good enough theory to explain the observed facts." [42]. The force of this argument lies in the fact that a multiverse proposal can be used to explain virtually anything, so it has almost no predictive power. I return to this issue in a moment.

Fourthly, the results obtained in this way are completely dependent on the measure used. Different (equally plausible) ways of assigning probabilities to candidate universes lead to totally different anthropic predictions. For example, Trotta and Starkman [47] present an explicit example based on the total number of possible observations observers can carry out, and conclude that in absence of a fundamental motivation for selecting one weighting scheme over another, the anthropic principle cannot be used to explain the value of $\Lambda$. The issue of the measure in a multiverse is unresolved, and is the subject of an ongoing debate. Related to this, anthropic bounds obtained on physical constants depend on the specific choice of the theory. For example, Ref. [32] claims that you can have a life-supporting universe (with carbon-12) without the weak interactions.

Finally, the physical existence or not of a multiverse is basically untestable [27,28], but is an essential component in the explanatory use of the SAP; so it is not clear if this can truly be regarded as a scientific theory, rather than a philosophical principle. The issue is discussed in [7,31]. Despite its unobservability, it is strongly supported because of the powerful explanatory framework it provides. However it is debatable whether it fits William of Ockham's stricture that "entities must not be multiplied beyond necessity"; one is postulating perhaps $10^{500}$ unobservable entities to explain the single entity we can observe. A principle of parsimony applies only if one can conclusively demonstrate the "necessity" part of the stricture.

Given these criticisms, how does it fare as regards the acid test: Can it make new predictions, or only retrospectively justify known relationships? There are two cases where this kind of reasoning may be claimed to have made new predictions, rather than post hoc justifying relations that we already knew.

Firstly, a remarkable pioneering paper by Fred Hoyle made a crucial observation regarding nuclear reactions required to create carbon-12 in helium-burning red giant stars $[34,35]$. In order to create the observed abundance of carbon, the triple-alpha process required an excited resonant state of carbon-12 with an energy level of $7.68 \mathrm{Mev}$ ([35], p. 134). The existence of the Hoyle state was experimentally confirmed shortly after by Willie Fowler; new data on the state is still being obtained today [17]. Since carbon is crucial to the existence of life as we know it, and indeed probably for existence of any physical living beings (because there seems to be no alternative to polypeptide chains for creating molecules of the required complexity), one can legitimately regard

\footnotetext{
4 This and other useful quotes are given in an extensive article on the anthropic principle in Wikipedia.
} 
existence of Hoyle's energy level as an anthropic requirement. Hoyle himself did not make that link at the time of his discovery, but seems to have done so later [38]; one can claim that anthropic requirements predict existence of this state. This is an instance of use of SAP to make a prediction, as originally proposed by Carter in his 1974 paper. However it has since been related to values of the fine structure constant [26]; this enables proposal of an anthropic explanation of the nature of the Hoyle state.

Secondly, an intriguing line of argument by Weinberg and collaborators [41] is that, due to a 'Principle of Mediocrity' espoused by Vilenkin, $\Lambda$ is not likely to be much smaller than the limit allowing galaxies to form. On this basis they concluded that if $\Omega_{\Lambda}$ turned out to be much less than 0.6 , anthropic reasoning could not explain why it was so small. This was at a time when work by Efstathiou and others suggested that $\Lambda$ was positive, but the definitive supernova and CMB data putting the value at $\Omega_{\Lambda} \simeq 0.7$ was not yet available. This almost counts as an anthropic prediction of something as yet unknown (in reviewing this argument more recently [53], Weinberg states that the observed value is still a bit low relative to this anthropic prediction). This is an example of use of SAP as an explanatory principle, but depends on the additional assumption of the Principle of Mediocrity - an unverifiable philosophical principle.

Taken together, these two cases show that the anthropic principle can potentially provide new predictions that can be experimentally tested. It is thus not always simply philosophy or tautology. However one must be cautious here: agreement with such predictions is supporting evidence for the multiverse hypothesis as an explanation, but does not amount to a proof that it is correct. If hypothesis $M$ (existence of a multiverse) leads to prediction $L$ (some range of values for $\Lambda$ ), it is simple logic that observation of $L$ does not necessarily imply validity of $M$ (this is only true if there is no other possible cause $M 1$ that also implies $L$ ). Furthermore, because this is only a probabilistic claim based on a further philosophical assumption, (not $L$ ) does not imply (not $M$ ): indeed no value of the cosmological constant can either prove or disprove existence of a multiverse, which is the crucial element in use of the anthropic principle for explanatory purposes. Nevertheless the Weinberg argument certainly makes it more plausible: it provides legitimate supporting evidence.

The need for the strong version of the anthropic principle would drop away if ever fundamental physics attained a foundational theory uniquely predicting the correct values of all the fundamental constants (which do of course allow life to exist) [12]. But this would not solve the anthropic mystery, as some have claimed [36], rather it would deepen it by changing its locus to a more fundamental level. What possible explanation could one then give for why theoretical physics principles (variational principles, specific symmetry groups, etc) would necessarily lead to the existence of life? It is not clear how the issue could be tackled then, even in principle. We are however apparently saved from this dilemma because our best approach to developing a fundamental theory of all forces (M-Theory) is presently heading in the opposite direction, by predicting more and more possible quantum vacua and associated effective theories $[44,45]$. The needed uniqueness is eluding us.

The anthropic principle continues to be the subject of much controversy today, some regarding it as a crucial foundational principle for cosmological argumentation, and others as giving up on science. The debate continues. Its current application as an 
explanatory principle is intimately tied in with the multiverse hypothesis. As this is observationally untestable, one can argue that it is a philosophical rather than strictly scientific proposal; but as such, it is a very productive hypothesis, leading to many interesting investigations. It deals with deep issues, and it will not go away.

A final comment: despite what is often claimed by its proponents, the anthropic principle used in this way does not solve issues of ultimate explanation; it only postpones them. If this hypothesis is indeed true, this simply leads to the next step in the argument: Why this particular multiverse? Why any multiverse? How do the 'laws' governing the multiverse arise? Where do they reside? And why are their governing constants what they are?

Carter's role in all of this was crucial: the paper [12] reprinted here initiated the vibrant discussion on this topic as a scientific as opposed to purely philosophical enterprise. However as is often the case, ${ }^{5}$ after a relatively short while it was replaced as a primary reference by other publications, and particularly (as is evidenced by the citation figures quoted at the start of this review essay) by the Barrow and Tipler book [3]. Carter himself moved on to discuss biological issues and the probability of life in the universe, stating [11] "The evidence suggests the evolutionary chain included at least one but probably not more than two links which were highly improbable (a priori) in the time available". He regarded this as an important result: in his 2004 review [12], he states "The example that seems to me most important was provided by the prediction [11] that the occurrence of anthropic observers would be rare, even on environmentally favorable planets such as ours". Hence it is not enough to talk about carbon or galaxies as being equivalent to the existence of observes (humans); one needs to look at biological issues. One specific issue here is whether the anthropic principle solves the crucial unsolved problem of how the DNA code was created. It has been claimed by Eugene Koonin [37] that it can do so in the context of eternal inflation because of the infinities proposed in that theory, but this is a controversial claim because those infinities are never realised in a finite time [29].

Carter later formulated an anthropic interpretation of quantum theory based on a microanthropic principle $[13,14]$ relating to the foundations of quantum physics. This has not made the same impact as the other versions. He is currently working on issues to do with the future and past of life on earth, considered in the light of anthropic measures of hominid and other terrestrial species [15].

Acknowledgments I thank Jean-Philippe Uzan, Jean-Pierre Lasota-Hirszowicz, Brandon Carter, and Martin Rees for helpful comments.

A brief biography of Brandon Carter was printed together with another Golden Oldie in Gen. Relativ. Gravit. 41, 2870-2871 (2009), doi:10.1007/s10714-009-0887-6.

\section{References}

1. Balashov, Y.V.: Resource letter AP-1: the anthropic principle. Am. J. Phys. 59, 1069 (1991)

2. Barrow, J.D.: The Constants of Nature; From Alpha to Omega-The Numbers that Encode the Deepest Secrets of the Universe. Pantheon Books, NY (2002)

\footnotetext{
5 Einstein's article "The Foundation of The General Theory of Relativity" in Annalen der Physik 49 (1916) has only 246 citations recorded in Google Scholar and 320 according to SPIRES.
} 
3. Barrow, J.D., Tipler, F.J.: The Anthropic Cosmological Principle. Oxford University Press, Oxford, ISBN 0-19-282147-4 (1986)

4. Bondi, H.: Cosmology. Cambridge University Press, Cambridge (1960)

5. Bostrom, N.: Anthropic Bias: Observation selection effects in Science and Philosophy. Routlege, New York (2002)

6. Carr, B. (ed.): Universe or Multiverse? Cambridge University Press, Cambridge (2007)

7. Carr, B., Ellis, G.F.R.: Universe or multiverse? Astron. Geophys. 49, 2.29-2.33 (2008)

8. Carter, B.: The significance of numerical coincidences in nature. Preprint (1967). Commented version available at arXiv:0710.3543v1

9. Carter, B.: Large Numbers in Astrophysics and Cosmology. Paper presented at Clifford Centennial Meet., Princeton (1970) unpublished

10. Carter, B.: Large number coincidences and the anthropic principle in cosmology. IAU Symposium 63: Confrontation of Cosmological Theories with Observational Data, pp. 291-298. Reidel, Dordrecht (1974)

11. Carter, B.: The anthropic principle and its implications for biological evolution. Philos. Trans. R. Soc. A 310, 347-363 (1983)

12. Carter, B.: Anthropic principle in cosmology. Contribution to Colloquium "Cosmology: facts and problems" (Coll'ege de France, June 2004) (2004) [arXiv:gr-qc/0606117v1]

13. Carter, B.: Anthropic interpretation of quantum theory. Contribution to 2003 Peyresq Physics Meeting "The Early Universe" (2004) [arXiv:hep-th/0403008]

14. Carter, B.: Micro-anthropic principle for quantum theory. In: Carr, B. (ed.) Universe or Multiverse? pp. 285-319. Cambridge University Press, Cambridge (2007) [arXiv:quant-ph/0503113v1]

15. Carter, B.: Anthropic measure of hominid (and other) terrestrials: http://luth.obspm.fr/luthier/carter/ trav/Finitude.pdf (2011)

16. Carr, B.J., Rees, M.J.: The anthropic principle and the structure of the physical world. Nature 278, 605-612 (1979)

17. Chernykh, M., Feldmeier, H., Neff, T., von Neumann-Cosel, P., Richter, A.: Pair decay width of the Hoyle state and carbon production in stars. Phys. Rev. Lett. 105, 022501 (2010) [arXiv:1004.3877]

18. Collins, C.B., Hawking, S.W.: Why is the universe isotropic? Astrophys. J. 180, 317-334 (1973)

19. Davies, P.: The Accidental Universe. Cambridge University Press, Cambridge (1982)

20. Demaret, J., Lambert, D.: Le Principe Anthropique.317-334 Armand Colin, Paris (1994)

21. Dicke, R.H.: Dirac's cosmology and Mach's Principle. Nature 192, 440-441 (1961)

22. Dirac, P.A.M.: The cosmological constants. Nature 139, 323 (1937)

23. Dirac, P.A.M.: A new basis for cosmology. Proc. R. Soc. Lond. A 165, 199-208 (1938)

24. Earman, J.: The SAP always rises: a critical examinaton of the anthropic principle. Am. Philos. Quart. 24, 307 (1987)

25. Eddington, A.S.: Fundamental Theory. Cambridge University Press, Cambridge (1948)

26. Ekstrom, S., Coc, A., Descouvemont, P., Meynet, G., Olive, K.A., Uzan, J.-P., Vangioni, E.: Effects of the variation of fundamental constants on Pop III stellar evolution (2009) arXiv:0911.2420v1

27. Ellis, G.F.R.: Issue in the philosophy of cosmology. In: Butterfield, J., Earman, J. (eds.) Handbook in Philosophy of Physics, pp. 1183-1285. Elsevier, Amsterdam (2006) [arXiv:astro-ph/0602280]

28. Ellis, G.F.R., in: Carr, B. (ed.): Universe or Multiverse? Cambridge University Press, Cambridge (2007)

29. Ellis, G.F.R., Stoeger, W.R.: A note on infinities in eternal inflation. Gen. Relativ. Gravit. 41, 1475-1484 (2009) [arXiv:1001.4590]

30. Gardner, M.: WAP, SAP, PAP, and FAP. New York Review of Books 23(8), 22-25 (1986)

31. Green, B.: The Hidden Reality: Parallel Universes and the Deep Laws of the Cosmos. Knopf, NY (2011)

32. Harnik, R., Kribs, G.D., Perez, G.: A universe without weak interactions. Phys. Rev. D 74, 035006 (2006) [arXiv:hep-ph/0604027]

33. Hogan, C.: Why is the universe just so? Rev. Mod. Phys. 72, 1149-1161 (2000)

34. Hoyle, F., Dunbar, D.N.F., Wenzel, W.A., Whaling, W.: A state in C 12 predicted from astrophysical evidence. Phys. Rev. 92, 1095-1161 (1953)

35. Hoyle, F.: On nuclear reactions occuring in very hot stars. I. The synthesis of elements from carbon to nickel. Ap. J. Suppl. 1, 121 (1954)

36. Kane, G.L., Perry, M.J., Zytkow, A.N.: The beginning of the end of the anthropic principle. New Astron. 7, 45-53 (2000) [arXiv:astro-ph/0001197v2] 
37. Koonin, E.: The cosmological model of eternal inflation and the transition from chance to biological evolution in the history of life. Biology Direct, open source access: http://www.biology-direct.com/ content/pdf/1745-6150-2-15.pdf (2007)

38. Kragh, H.: When is a prediction anthropic? Fred Hoyle and the $7.68 \mathrm{MeV}$ Resonance http:// philsci-archive.pitt.edu/5332/1/3alphaphil.pdf

39. Leslie, J.: Universes. Routledge, London (1989)

40. Linde, A.D.: Eternally existing self-reproducing chaotic inflationary universe. Phys. Lett. B 175, 395-400 (1986)

41. Martel, H., Shapiro, P.R., Weinberg, S.: Likely values of the cosmological constant. Astrophys. J. 492, 29 (1998) [arXiv:astro-ph/9701099v1]

42. Penrose, R.: The Emperor's New Mind (Chapter 10). Oxford University Press, Oxford (1989)

43. Rees, M.J.: Just Six Numbers: The Deep Forces That Shape the Universe. Weidenfeld and Nicolson, London (2000)

44. Susskind, L.: The anthropic landscape of string theory. arXiv:hep-th/0302219 (2003)

45. Susskind, L.: The cosmic landscape: string theory and the illusion of intelligent design. (Little, Brown, 2005) (2005)

46. Tegmark, M.: Is 'the theory of everything' merely the ultimate ensemble theory? Ann. Phys. 270, 1-51 (1998)

47. Trotta, R., Starkman, G.D.: Why anthropic reasoning cannot predict? Phys. Rev. Lett. 97, 201301 -51 (2006)

48. Uzan, J.-P.: The fundamental constants and their variation: observational status and theoretical motivations. Rev. Mod. Phys 75, 403-51 (2003)

49. Uzan, J.-P.: Varying constants, gravitation and cosmology. Living Rev. Relativ. (2011) [arXiv: 1009.5514$]$

50. Wallace, A.R.: Man's Place in the Universe: A Study of the Results of Scientific Research in Relation to the Unity or Plurality of Worlds, 4th edn. pp. 256-7. George Bell, London (1904)

51. Weinberg, S.: Anthropic bound on the cosmological constant. Phys. Rev. Lett. 59, 2607-2610 (1987)

52. Weinberg, S.: The cosmological constant problem. Rev. Mod. Phys. 61, 1-23 (1989)

53. Weinberg, S.: Living in the multiverse. In: Carr, B. (ed.) Universe or Multiverse? pp. 29-42. Cambridge University Press, Cambridge (2007) [arXiv:hep-th/0511037] 